OHSTPY-HEP-T-03-001

February 2003

\title{
A Natural Framework for Bi-large Neutrino Mixing
}

\author{
S. Raby \\ Department of Physics, The Ohio State University, \\ 174 W. 18th Ave., Columbus, Ohio 43210
}

\begin{abstract}
In this letter we present a "natural" framework for obtaining bi-large neutrino mixing incorporating the Frampton-Glashow-Yanagida neutrino mass matrix ansatz. We show that an $S U(2) \times U(1)$ family symmetry can provide the desired FGY neutrino mass ansatz in a minimal supersymmetric standard model. We also show how to obtain an approximate FGY ansatz in an $S O(10)$ SUSY GUT. In this context, the same $S U(2) \times U(1)$ family symmetry also generates the hierarchy of fermion masses as well as ameliorating SUSY flavor problems.
\end{abstract}


In a recent paper [1] [FGY] a simple ansatz for neutrino masses has been presented which has several significant virtues. It is an elegant framework for so-called bi-large neutrino mixing, i.e. giving maximal $\nu_{\mu}-\nu_{\tau}$ mixing for atmospheric neutrinos and large mixing angle Mikheyev-Smirnov-Wolfenstein mixing for solar neutrinos. ${ }^{1}$ In addition, the sign of the cosmological baryon asymmetry is related to $\mathrm{CP}$ violation which may be observable in low energy neutrino oscillation experiments. ${ }^{2}$ In this letter we present a simple $S U(2) \times U(1)$ symmetry within the context of supersymmetry which makes the FGY ansatz "natural". We then extend this simple realization to an $S O(10)$ grand unified theory [GUT] which also fits charged fermion masses and mixing angles. In the latter example, the possibility of additional $\mathrm{CP}$ violating angles destroys the leptogenesis/neutrino CP violation connection, but still provides a natural framework for bi-large neutrino mixing.

Consider the three lepton doublets $l_{i}, i=1,2,3$. Under an $S U(2)$ family symmetry, two of the lepton doublets transform as a doublet given by $L_{a}=\left(\begin{array}{l}l_{1} \\ l_{2}\end{array}\right)$, while $l_{3}$ is a singlet. In addition, we require two flavor (anti)-doublets $\phi^{a}$ and $\tilde{\phi}^{a}$, 4 flavor singlets $N_{1}, N_{2}, S_{1}, S_{2}$ and the standard model Higgs doublet $H$.

The superpotential is given by

$$
W=\frac{H}{M}\left(L_{a} \phi^{a} N_{1}+L_{a} \tilde{\phi}^{a} N_{2}+l_{3} \omega N_{2}\right)+\frac{1}{2}\left(S_{1} N_{1}^{2}+S_{2} N_{2}^{2}\right)
$$

where $M$ is some large mass scale. Note arbitrary order one dimensionless couplings are implicitly assumed to multiply the terms in $W$. In addition, we assume that $W$ is CP invariant where $\mathrm{CP}$ is presumed to be spontaneously violated in complex vevs. The $U(1)$ charge assignments for the fields $\left\{L_{a}, l_{3}, N_{1}, N_{2}, \phi^{a}, \tilde{\phi}^{a}, \omega, S_{1}, S_{2}\right\}$ are as follows $\{1, \alpha, x, y,-(x+1),-(y+1),-(\alpha+y),-2 x,-2 y\}$ with $x \neq y$.

We assume $\phi, \tilde{\phi}$ get vevs $\langle\phi\rangle=\left(\begin{array}{c}\phi^{1} \\ \phi^{2}\end{array}\right)$ and $\langle\tilde{\phi}\rangle=\left(\begin{array}{c}0 \\ \tilde{\phi}^{2}\end{array}\right)$. This does not require fine tuning since any arbitrary vevs can be rotated into this direction using an $S U(2) \times U(1)$ flavor rotation. The vevs $\left\langle S_{i}\right\rangle=M_{i}, i=1,2$ are also needed to give the states $N_{i}$ a large see-saw mass. These vevs can be obtained via suitable terms added to the superpotential. We then obtain the $3 \times 2$ mixing matrix $D^{t r}$ defined by

$$
\left(\begin{array}{lll}
\nu_{1} & \nu_{2} & \nu_{3}
\end{array}\right) D^{t r}\left(\begin{array}{l}
N_{1} \\
N_{2}
\end{array}\right) \equiv\left(\begin{array}{lll}
\nu_{1} & \nu_{2} & \nu_{3}
\end{array}\right)\left(\begin{array}{cc}
a & 0 \\
a^{\prime} e^{i \delta} & b \\
0 & b^{\prime}
\end{array}\right)\left(\begin{array}{c}
N_{1} \\
N_{2}
\end{array}\right)
$$

where

$$
a=v \sin \beta \frac{\phi^{1}}{\sqrt{2} M}, \quad a^{\prime} e^{i \delta}=v \sin \beta \frac{\phi^{2}}{\sqrt{2} M}, \quad b=v \sin \beta \frac{\tilde{\phi}^{2}}{\sqrt{2} M}, \quad b^{\prime}=v \sin \beta \frac{\omega}{\sqrt{2} M}
$$

\footnotetext{
${ }^{1}$ For other recent discussions on similar frameworks for bi-large mixing, see Refs. $[2,3]$

${ }^{2}$ For some recent discussions of leptogenesis and low energy physics, see Refs. [4]-[8].
} 
and $\langle H\rangle=\left(\begin{array}{c}0 \\ v \sin \beta / \sqrt{2}\end{array}\right)$. Note, the vevs are in general complex, however, with phase redefinitions of the fields $N_{1}, N_{2}, l_{1}, l_{2}, l_{3}$ all phases but $\delta$ can be transformed away. As we show later, this is consistent with the charged lepton sector.

The neutrino masses are given by

$$
\mathcal{M}_{F G Y}=D^{t r} M_{N}^{-1} D
$$

with

$$
M_{N}=\left(\begin{array}{cc}
M_{1} & 0 \\
0 & M_{2}
\end{array}\right) .
$$

This is exactly the FGY ansatz [1]. The diagonalized neutrino mass matrix is given by

$$
\mathcal{M}^{D}=U_{F G Y}^{t r} \mathcal{M}_{F G Y} U_{F G Y}
$$

where

$$
U_{F G Y}=\left(\begin{array}{ccc}
1 / \sqrt{2} & 1 / \sqrt{2} & 0 \\
-1 / 2 & 1 / 2 & 1 / \sqrt{2} \\
1 / 2 & -1 / 2 & 1 / \sqrt{2}
\end{array}\right) \times\left(\begin{array}{ccc}
1 & 0 & 0 \\
0 & \cos \theta & \sin \theta \\
0 & -\sin \theta & \cos \theta
\end{array}\right)
$$

is the FGY neutrino mixing matrix (for the case $b=b^{\prime}$ and $a^{\prime}=\sqrt{2} a$ ). The three neutrino mass eigenvalues are given by

$$
m_{\nu_{3}} \approx 2 b^{2} / M_{2} \gg m_{\nu_{2}} \approx 2 a^{2} / M_{1}, \quad m_{\nu_{1}}=0
$$

and the small angle $\theta \sim m_{\nu_{2}} /\left(\sqrt{2} m_{\nu_{3}}\right)$. Recall, in order to fit neutrino data we have

$$
\begin{aligned}
& m_{\nu_{3}} \approx 0.05 \mathrm{eV}=\sqrt{\Delta m_{a t m}^{2}} \\
& m_{\nu_{2}} \approx 7 \times 10^{-3} \mathrm{eV}=\sqrt{\Delta m_{\text {sol }}^{2}}
\end{aligned}
$$

In order to complete the discussion of neutrino masses and mixing angles, it is necessary to discuss the charged lepton mass matrix. If this matrix is to a good approximation diagonal, then the neutrino mass matrix above may be considered to be in a lepton flavor basis and our results are unaffected by the charged lepton sector. There are many possible choices for the $S U(2) \times U(1)$ assignments of the right handed charged leptons. In order to be more specific we shall discuss an example in which the charged leptons are $S U(2)$ singlets. Consider the superpotential

$$
W_{\text {ch.leptons }}=\frac{\bar{H}}{M}\left(L_{a} \phi^{a} \bar{e}_{1}+L_{a} \tilde{\phi}^{a} \bar{e}_{2}+l_{3}\left(\omega \bar{e}_{2}+\bar{\omega} \bar{e}_{3}\right)\right)
$$

where

$$
\begin{gathered}
\bar{a}=v \cos \beta \frac{\phi^{1}}{\sqrt{2} M}, \quad \bar{a}^{\prime} e^{i \delta}=v \cos \beta \frac{\phi^{2}}{\sqrt{2} M}, \quad \bar{b}=v \cos \beta \frac{\tilde{\phi}^{2}}{\sqrt{2} M}, \\
\bar{b}^{\prime}=v \cos \beta \frac{\omega}{\sqrt{2} M}, \quad \bar{c}=v \cos \beta \frac{\bar{\omega}}{\sqrt{2} M} .
\end{gathered}
$$


and $\langle\bar{H}\rangle=\left(\begin{array}{c}v \cos \beta / \sqrt{2} \\ 0\end{array}\right)$. The $U(1)$ charge assignments for the fields $\left\{\bar{e}_{1}, \bar{e}_{2}, \bar{e}_{3}, \bar{\omega}\right\}$ are as follows $\{x, y, z,-(\alpha+z)\}$. Note, with suitable phase redefinitions of the fields $\bar{e}_{i}, i=1,2,3$, all phases but $\delta$ may be transformed away.

The charged lepton mass matrix is then given by (defined with left-handed doublets on the right)

$$
m_{l}=\left(\begin{array}{ccc}
\bar{a} & \bar{a}^{\prime} e^{i \delta} & 0 \\
0 & \bar{b} & \bar{b}^{\prime} \\
0 & 0 & \bar{c}
\end{array}\right)
$$

Note, using the constraint from the neutrino sector, we have $\bar{a} \approx \bar{a}^{\prime}, \bar{b} \approx \bar{b}^{\prime}$ and we take $\bar{a}, \bar{a}^{\prime} \ll \bar{b}, \bar{b}^{\prime} \ll \bar{c}$. Hence

$$
m_{e} \approx \bar{a}, \quad m_{\mu} \approx \bar{b}, \quad m_{\tau} \approx \bar{c}
$$

and the charged lepton mass matrix is approximately diagonal. The matrix can be diagonalized by a left-handed (right-handed) unitary transformation $U_{e}=\mathcal{P} V_{e}\left(U_{\bar{e}}=\right.$ $\left.\mathcal{P} V_{\bar{e}}\right)$ with $m_{l}^{\text {diagonal }}=U_{\bar{e}}^{\dagger} m_{l} U_{e}$ where $V_{e}\left(V_{\bar{e}}\right)$ are real orthogonal matrices and $\mathcal{P}$ is a diagonal phase matrix. We have

$$
U_{e} \approx\left(\begin{array}{ccc}
1 & 0 & 0 \\
0 & e^{-i \delta} & 0 \\
0 & 0 & -e^{-i \delta}
\end{array}\right) \times\left(\begin{array}{ccc}
1 & \bar{a}^{2} / \bar{b}^{2} & 0 \\
-\bar{a}^{2} / \bar{b}^{2} & 1 & -\bar{b}^{2} / \bar{c}^{2} \\
0 & \bar{b}^{2} / \bar{c}^{2} & 1
\end{array}\right) \approx V_{e} \mathcal{P}
$$

where $V_{e}$ is approximately the $3 \times 3$ unit matrix with negligible mixing angles. In the lepton flavor basis we have

$$
\mathcal{M}=U_{e}^{t r}\left[D^{t r} M_{N}^{-1} D\right] U_{e} \approx \mathcal{M}_{F G Y}
$$

where with a suitable phase redefinition of the lepton doublets and singlets we recover the FGY ansatz.

It is clear that we have obtained the FGY ansatz as a consequence of a spontaneously broken $S U(2) \times U(1)$ family symmetry. Note that the FGY ansatz has a discrete ambiguity related to the mass ordering of the heavy Majorana neutrinos. It has been shown [7] that leptogenesis and the observed baryon asymmetry correlates this ambiguity with the sign of the $\mathrm{CP}$ violating phase $\delta$ such that for $M_{1} \ll M_{2} \quad(\delta>0)$ or for $M_{1} \gg M_{2} \quad(\delta<0)$. These two possibilities also lead to different predictions for low energy lepton flavor violation [7]. This ambiguity is resolved in our model since now (using Eqns. 8 and 14) we have $\left(m_{e} / m_{\mu}\right)^{2} \approx(\bar{a} / \bar{b})^{2} \approx(a / b)^{2} \approx\left(M_{1} / M_{2}\right)\left(m_{\nu_{2}} / m_{\nu_{3}}\right)$. Hence $\left(M_{1} / M_{2}\right) \sim 10^{-3}$. We thus have the predictions for the lepton flavor violating branching ratios [7]:

$$
\begin{aligned}
& B(\mu \rightarrow e \gamma) \approx 2 r 10^{-13} \\
& B(\tau \rightarrow \mu \gamma) \geq 3 r 10^{-12}
\end{aligned}
$$

with $r \approx(\tan \beta / 10)^{2}\left(150 \mathrm{GeV} / m_{S U S Y}\right)^{4}$. 
Note, the FGY ansatz can accomodate bi-maximal mixing but it cannot explain why $a \sim a^{\prime}$ or $b \sim b^{\prime}$. This difficulty, however, is somewhat reduced in our $S O(10)$ example. In addition, note this model has a straightforward generalization to $S U(5)$ with the conversions of $L_{a}, l_{3} \rightarrow \overline{5}_{a}, \overline{5}_{3} ; \bar{e}_{i} \rightarrow 10_{i}, i=1,2,3$ and the Higgs doublets $H \rightarrow 5_{H}, \bar{H} \rightarrow \overline{5}_{H}$. Of course we would then need to discuss quark masses and resolve the problem of the simple GUT scale $S U(5)$ relations $-\lambda_{d}=\lambda_{e}, \lambda_{s}=\lambda_{\mu}$. However, we will not consider an $S U(5)$ extension further here. Instead we consider a simple extension to $S O(10)$. However before we discuss an $S O(10)$ model, let us consider the family symmetry $S U(2) \times U(1)$ further and possible family symmetry breaking mechanisms. Perhaps the first question to address is whether the family symmetry is local or global. If, however, we assume that the scale of symmetry breaking $M$ is sufficiently large with $M>>M_{Z}$, then either way it will not have any observable low energy consequences. If it is a global symmetry, the massless Nambu-Goldstone bosons will decouple from low energy physics as $1 / M$, whereas if it is a local symmetry the new $S U(2) \times U(1)$ gauge bosons will get mass of order $M$, hence they also decouple from the low energy physics. On the other hand, the question of whether it is a local or global symmetry will affect the symmetry breaking mechanisms. Consider for example that it is a local symmetry. Then in order to find D flat directions, we may need to include conjugate $S U(2)$ doublet flavon fields $\phi^{c}, \tilde{\phi}^{c}$ with $U(1)$ charges $(x+1),(y+1)$. These then get non-zero vevs in the $\mathrm{D}$ flat directions. In addition, if the $U(1)$ has a non-vanishing trace, it will obtain a Fayet-Iliopoulos D term of order the effective cut-off scale of the theory. This might give these flavon fields a supersymmetry preserving vev at this scale.

Now consider a similar realization of the same symmetry in an $S O(10)$ SUSY GUT with an $S U(2) \times U(1)$ family symmetry. ${ }^{3}$ The three families of quarks and leptons are contained in three 16 dimensional representations of $S O(10)\left\{16_{a}, 16_{3}\right\}$ with $16_{a}$, a = 1,2 an $S U(2)$ flavor doublet. Consider the charged fermion sector first. Although the charged fermion sector is not the main focus of this letter it is necessary to present a possible superpotential which results in charged fermion masses and mixing angles, since in $S O(10)$ the neutrino and charged fermion sectors are inextricably intertwined.

The superpotential resulting in charged fermion masses and mixing angles is given by

$$
\begin{aligned}
& W_{\text {ch.fermions }}=\quad 16_{3} 1016_{3}+16_{a} 10 \chi^{a} \\
& +\bar{\chi}_{a}\left(M_{\chi} \chi^{a}+45 \frac{\phi^{a}}{\bar{M}} 16_{3}+45 \frac{\tilde{\phi}^{a} \tilde{\phi}^{b}}{(\hat{M})^{2}} 16_{b}+A^{a b} 16_{b}\right)
\end{aligned}
$$

where $M_{\chi}=M_{0}(1+\alpha X+\beta Y)$ includes $S O(10)$ breaking vevs in the $X$ and $Y$ directions, $\phi^{a}, \tilde{\phi}^{a}, A^{a b}=-A^{b a}$ are $S O(10)$ singlet flavon fields with explicit $S U(2)$ properties, $\hat{M}$ is an $S O(10)$ singlet mass and $\langle 45\rangle \sim(B-L) M_{G}$. The fields $\phi, \tilde{\phi}$ are assumed to obtain vevs

$$
\langle\phi\rangle=\left(\begin{array}{c}
\phi^{1} \\
\phi^{2}
\end{array}\right),\langle\tilde{\phi}\rangle=\left(\begin{array}{c}
0 \\
\tilde{\phi}^{2}
\end{array}\right) .
$$

\footnotetext{
${ }^{3}$ For related charged fermion analyses in $S O(10)$ SUSY GUTS with $S U(2) \times U(1)^{n}$ (or $D_{3}$ ) family symmetries, see $[9,10]$ (or [11]).
} 
Note, once again, this is completely general, since arbitrary vevs can be rotated into this direction by the $S U(2) \times U(1)$ family symmetry.

The superpotential, (Eqn. 18) results in the following charged fermion Yukawa matrices: ${ }^{4}$

$$
\begin{aligned}
& Y_{u}=\left(\begin{array}{ccc}
0 & \epsilon^{\prime} \rho & -\epsilon \xi \\
-\epsilon^{\prime} \rho & \tilde{\epsilon} \rho & -\epsilon \\
\epsilon \xi & \epsilon & 1
\end{array}\right) \lambda \\
& Y_{d}=\left(\begin{array}{ccc}
0 & \epsilon^{\prime} & -\epsilon \xi \sigma \\
-\epsilon^{\prime} & \tilde{\epsilon} & -\epsilon \sigma \\
\epsilon \xi & \epsilon & 1
\end{array}\right) \lambda \\
& Y_{e}=\left(\begin{array}{ccc}
0 & -\epsilon^{\prime} & 3 \epsilon \xi \\
\epsilon^{\prime} & 3 \tilde{\epsilon} & 3 \epsilon \\
-3 \epsilon \xi \sigma & -3 \epsilon \sigma & 1
\end{array}\right) \lambda
\end{aligned}
$$

with

$$
\begin{aligned}
\xi & =\phi^{1} / \phi^{2} ; \quad \tilde{\epsilon} \propto\left(\tilde{\phi}^{2} / \hat{M}\right)^{2} ; \\
\epsilon & \propto \phi^{2} / \hat{M} ; \quad \epsilon^{\prime} \sim\left(A^{12} / M_{0}\right) ; \\
\sigma & =\frac{1+\alpha}{1-3 \alpha} ; \quad \rho \sim \beta \ll \alpha .
\end{aligned}
$$

It has been shown in Ref. [10] that excellent fits to charged fermion masses and mixing angles are obtained with this Yukawa structure.

In the three $16 \mathrm{~s}$ we have three electroweak doublet neutrinos $\left(\nu_{a}, \nu_{3}\right)$ and three electroweak singlet anti-neutrinos $\left(\bar{\nu}_{a}, \bar{\nu}_{3}\right) .{ }^{5}$ The superpotential $W_{\text {ch.fermions }}$ also results in a neutrino Yukawa matrix:

$$
Y_{\nu}=\left(\begin{array}{ccc}
0 & -\epsilon^{\prime} \omega & \frac{3}{2} \epsilon \xi \omega \\
\epsilon^{\prime} \omega & 3 \tilde{\epsilon} \omega & \frac{3}{2} \epsilon \omega \\
-3 \epsilon \xi \sigma & -3 \epsilon \sigma & 1
\end{array}\right) \lambda
$$

with $\omega=2 \sigma /(2 \sigma-1)$ and a Dirac neutrino mass matrix given by

$$
m_{\nu} \equiv Y_{\nu} \frac{v}{\sqrt{2}} \sin \beta
$$

In addition, the anti-neutrinos get GUT scale masses by mixing with three $S O(10) \times$ $S U(2)$ singlets $\left\{N_{i}, i=1,2,3\right\}$. The full superpotential is given by $W=W_{\text {ch.fermions }}+$ $W_{\text {neutrino }}$ with

$$
\begin{gathered}
W_{\text {neutrino }}=\frac{\overline{16}}{\hat{M}}\left(N_{1} \tilde{\phi}^{a} 16_{a}+N_{2} \phi^{a} 16_{a}+N_{3} \theta 16_{3}\right) \\
+\frac{1}{2}\left(S_{1} N_{1}^{2}+S_{2} N_{2}^{2}\right)
\end{gathered}
$$

\footnotetext{
${ }^{4}$ Note, in this case the doublets are on the left.

${ }^{5}$ In an equivalent notation, we have three left-handed neutrinos $\left(\nu_{L a} \equiv \nu_{a}, \nu_{L 3} \equiv \nu_{3}\right)$ and three right-handed neutrinos defined by $\left(\nu_{R a} \equiv \bar{\nu}_{a}^{*}, \nu_{R 3} \equiv \bar{\nu}_{3}^{*}\right)$.
} 
and we assume $\overline{16}$ obtains a vev $v_{16}$ in the right-handed neutrino direction, $\left\langle S_{i}\right\rangle=M_{i}$ for $i=1,2$ and $\langle\theta\rangle=\theta$. We thus obtain the effective neutrino mass terms given by

$$
W=\nu m_{\nu} \bar{\nu}+\bar{\nu} V N+\frac{1}{2} N M_{N} N
$$

with

$$
V^{t r}=\frac{v_{16}}{\hat{M}}\left(\begin{array}{ccc}
0 & \tilde{\phi}^{2} & 0 \\
\phi^{1} & \phi^{2} & 0 \\
0 & 0 & \theta
\end{array}\right), M_{N}=\operatorname{diag}\left(M_{1}, M_{2}, 0\right)
$$

where $V^{t r}$ is the transpose of $V$. The family symmetry is at least $S U(2) \times U(1)$ where the $S U(2)$ charges are evident, while the $U(1)$ charge assignments for $\left\{\overline{16}, 16_{3}, 16_{a}\right.$, $\left.N_{1}, N_{2}, N_{3}, \phi^{a}, \tilde{\phi}^{a}, \theta, S_{1}, S_{2}\right\}$ are given by $\left\{-2 n_{1}+n_{2}-1,1, x, n_{1}, n_{2}, n_{3}\right.$, $\left.2\left(n_{1}-n_{2}\right)+1-x,\left(n_{1}-n_{2}\right)+1-x, 2 n_{1}-\left(n_{2}+n_{3}\right),-2 n_{1},-2 n_{2}\right\}$.

The electroweak singlet neutrinos $\{\bar{\nu}, N\}$ have large masses of order $V, M_{N} \sim M_{G}$. After integrating out these heavy neutrinos, we obtain the light neutrino mass matrix given by

$$
\mathcal{M}=U_{e}^{t r}\left[m_{\nu}\left(V^{t r}\right)^{-1} M_{N} V^{-1} m_{\nu}^{t r}\right] U_{e}
$$

It is explicitly defined in the lepton flavor basis where $U_{e}$ is the $3 \times 3$ unitary matrix for left-handed leptons needed to diagonalize $Y_{e}$ (eqn. 20), i.e. $Y_{e}^{D}=U_{e}^{t r} Y_{e} U_{\bar{e}}^{*}$.

Note,

$$
\left(V^{t r}\right)^{-1}=\frac{\hat{M}}{v_{16}}\left(\begin{array}{ccc}
-1 /\left(\tilde{\phi}^{2} \xi\right) & 1 / \phi^{1} & 0 \\
1 / \tilde{\phi}^{2} & 0 & 0 \\
0 & 0 & 1 / \theta
\end{array}\right)
$$

Let us now define

$$
D^{t r} \equiv m_{\nu}\left(V^{t r}\right)^{-1} M_{N} \mathcal{P}=\left(\begin{array}{cc}
a & 0 \\
a^{\prime} & b \\
0 & b^{\prime}
\end{array}\right)
$$

where

$$
\mathcal{P}=\left(\begin{array}{ll}
1 & 0 \\
0 & 1 \\
0 & 0
\end{array}\right)
$$

and $m_{\nu}$ is the Dirac neutrino mass (Eqn. 23). We then obtain

$$
\mathcal{M}=U_{e}^{t r}\left[D^{t r} \hat{M}_{N}^{-1} D\right] U_{e}
$$

with

$$
\hat{M}_{N} \equiv\left(\begin{array}{cc}
M_{1} & 0 \\
0 & M_{2}
\end{array}\right)
$$

The parameters $\left\{a, a^{\prime}, b, b^{\prime}\right\}$ are given by

$$
a \equiv \quad-\epsilon^{\prime} \omega \lambda\left(M_{1} / \tilde{\phi}^{2}\right) \frac{\hat{M}}{v_{16}} \frac{v \sin \beta}{\sqrt{2}}
$$




$$
\begin{array}{rlrl}
a^{\prime} & \equiv & & \left(-\epsilon^{\prime} \xi^{-1}+3 \tilde{\epsilon}\right) \omega \lambda\left(M_{1} / \tilde{\phi}^{2}\right) \frac{\hat{M}}{v_{16}} \frac{v \sin \beta}{\sqrt{2}} \\
b & \equiv & \epsilon^{\prime} \omega \lambda\left(M_{2} / \phi^{1}\right) \frac{\hat{M}}{v_{16}} \frac{v \sin \beta}{\sqrt{2}} \\
b^{\prime} & \equiv & -3 \epsilon \xi \sigma \lambda\left(M_{2} / \phi^{1}\right) \frac{\hat{M}}{v_{16}} \frac{v \sin \beta}{\sqrt{2}} .
\end{array}
$$

Note, these parameters are in general all complex. We have $b \sim b^{\prime}$, since $\epsilon^{\prime} \sim \phi^{1} / \hat{M} \sim$ $\epsilon \xi \sim 3 \tilde{\epsilon} \xi$ (see Eqns. 21 and 36). However, without fine-tuning, we find $a \ll a^{\prime}$. This can be remedied if the implicit order one coefficients in these expressions are used to fine tune the two terms in $a^{\prime}$ so that they cancel to about one part in 10 giving $a^{\prime} \sim a$. Finally, due to the small mixing angles in $U_{e}$, the observable neutrino mixing matrix $U$ used to diagonalize $\mathcal{M}$ via

$$
\mathcal{M}^{D}=U^{t r} \mathcal{M} U
$$

is given by

$$
U=U_{e}^{\dagger} U_{F G Y}
$$

where $U_{F G Y}$ is the FGY neutrino mixing matrix (Eqn. 7). Using the results of Blažek et al. (2000) (see Table 2 and discussion in Sect. 4.1) [10] we have

$$
\left|Y_{e}\right| \approx\left(\begin{array}{ccc}
0 & 0.003 & 0.004 \\
0.003 & 0.03 & 0.12 \\
0.004 & 0.12 & 1
\end{array}\right) 0.8
$$

which gives $\left|\left(U_{e}^{t r}\right)_{12}\right| \approx 0.16,\left|\left(U_{e}^{t r}\right)_{23}\right| \approx 0.12$ and $\left|\left(U_{e}^{t r}\right)_{13}\right| \approx 0.01$. Hence, the small mixing angles in $U_{e}$ do not significantly affect the result of bi-large mixing.

In the $S O(10)$ version of the theory, the discrete ambiguity related to the mass ordering of the heavy Majorana neutrinos is resolved with $M_{1} / M_{2} \sim 10^{3}$ where we have used the relation $m_{\nu_{2}} / m_{\nu_{3}} \approx\left(m_{e} / m_{\mu}\right)\left(M_{1} / M_{2}\right) \tilde{\epsilon}$. However since the heavy Majorana sector, including the fields $\left\{\bar{\nu}_{i} ; N_{i}\right.$, for $\left.i=1,2,3\right\}$ is much more complicated than studied previously [7], and because of the additional CP violating phases in this model, it is not clear what constraints may be derived by requiring that the observed cosmological density come via leptognesis.

However, the main point of FGY is that $\mathrm{CP}$ violation, as measured in low energy neutrino oscillation experiments or via the cosmological baryon asymmetry, is governed by the single phase $\delta$ given in the expression $a^{\prime} e^{i \delta}$ (Eqn. 3). In the minimal supersymmetric example we retain this nice feature. In the $S O(10)$ example, we lose this simple connection since there are generically additional CP violating angles present in $U_{e}$. Note, however, that now there is the possibility of relating $\mathrm{CP}$ violation in neutrino physics to $\mathrm{CP}$ violation in the CKM matrix.

In this letter we have presented a "natural" framework for the FGY [1] neutrino mass matrix ansatz. The FGY ansatz has two major virtues,

- it accomodates bi-large neutrino mixing, and

- it relates $\mathrm{CP}$ violation in neutrino oscillations with the sign of the cosmological baryon asymmetry. 
We show that an $S U(2) \times U(1)$ family symmetry can provide the desired FGY neutrino mass ansatz in a minimal supersymmetric standard model. Moreover the discrete ambiguity for the ratio of Majorana neutrino masses is resolved. This then makes definite predictions for the sign of the CP violating phase $\delta$, and also for lepton flavor violating processes (Eqn. 17). We have also shown how to obtain an approximate FGY ansatz in an $S O(10)$ SUSY GUT. In this latter example we lose the direct connection between high energy $\mathrm{CP}$ violation made visible by leptogenesis and direct $\mathrm{CP}$ violation observable in low energy neutrino oscillations. However since the neutrino sector is now intertwined with the charged fermions, we have other interesting relations. For example, the relation $b \approx b^{\prime}$, needed for a large atmospheric neutrino mixing angle, is now automatic. The large solar mixing angle is, however, still easily accomodated. Moreover, the same $S U(2) \times U(1)$ family symmetry has the virtue of generating the fermion mass hierarchy as well as suppressing large flavor changing neutral current processes [9, 10]. Finally, it is important to note that our results are not significantly affected by renormalization group [RG] running. It is well known that RG running of the effective dimension 5 lepton-Higgs interaction below the Majorana mass scales $\left[M_{1}, M_{2}\right]$ does not significantly affect the results for neutrino masses and mixing angles when we have a hierarchical neutrino spectrum[12].

\section{Acknowledgements}

Partial support for this work was obtained under DOE grant DOE/ER/01545-840 and also a grant from the Alexander von Humboldt Foundation. I would like to thank the faculty and staff at the Universität Bonn where this work was begun. I also greatly benefitted from discussions with P.H. Frampton, J.E. Kim, H.D. Kim, L. Schradin and K. Tobe.

\section{References}

[1] P. H. Frampton, S. L. Glashow and T. Yanagida, Phys. Lett. B 548, 119 (2002) [arXiv:hep-ph/0208157].

[2] R. Kuchimanchi and R. N. Mohapatra, Phys. Rev. D 66, 051301 (2002) [arXiv:hep$\mathrm{ph} / 0207110]$.

[3] S. F. King, arXiv:hep-ph/0211228.

[4] J. R. Ellis and M. Raidal, Nucl. Phys. B 643, 229 (2002) [arXiv:hep-ph/0206174].

[5] T. Endoh, S. Kaneko, S. K. Kang, T. Morozumi and M. Tanimoto, Phys. Rev. Lett. 89, 231601 (2002) [arXiv:hep-ph/0209020].

[6] W. Buchmuller, P. Di Bari and M. Plumacher, Phys. Lett. B 547, 128 (2002) [arXiv:hep-ph/0209301].

[7] M. Raidal and A. Strumia, Phys. Lett. B 553, 72 (2003) [arXiv:hep-ph/0210021]. 
[8] G. C. Branco, R. Gonzalez Felipe, F. R. Joaquim, I. Masina, M. N. Rebelo and C. A. Savoy, arXiv:hep-ph/0211001.

[9] R. Barbieri, L. J. Hall, S. Raby and A. Romanino, Nucl. Phys. B 493, 3 (1997) [arXiv:hep-ph/9610449].

[10] T. Blazek, S. Raby and K. Tobe, Phys. Rev. D 60, 113001 (1999) [arXiv:hepph/9903340]; Phys. Rev. D 62, 055001 (2000) [arXiv:hep-ph/9912482].

[11] R. Dermisek and S. Raby, Phys. Rev. D 62, 015007 (2000) [arXiv:hep-ph/9911275].

[12] P. H. Chankowski and S. Pokorski, Int. J. Mod. Phys. A 17, 575 (2002) [arXiv:hep$\mathrm{ph} / 0110249]$ and references therein. 